(Aus der Zoologischen Station Büsum, Nordsee.)

\title{
Beiträge zur Physiologie des Farbenwechsels der Fische.
}

\section{Untersuchungen an Pleuronectiden.}

Von

\section{J. Georg Schaefer, Köln.}

Mit 16 Textabbildungen.

Nach gemeinsam mit Herrn Theod or Müller (zur Zeit Physiol. Institut Genf) ausgeführten Versuchen.

(Eingegangen am 29. November 1920.)

1. Einleitung. (S. 25).

Inhalt :

2. Bau der Chromatophoren von Pleuronectes platessa. (S. 27).

3. Allgemeines ihbr Verhalten gegen Reize. (S. 28).

4. Beeinflussung der Chromatophoren durch das Nervensystem. (S. 31).

5. Giftwirkung und Farbenwechsel. (S. 36).

6. Zusammenfassung und Schluß. (S. 44).

\section{Einleitung.}

Die Sicherheit der anatomischen Ergebnisse über die Elemente des Farbenwechsels der Fische steht in keinem Verhältnis zu der der physiologischen Beobachtungen. Während über Anordnung, Bau und Entwicklungsgeschichte der Chromatophoren, über die Pigmentbildung, über die Frage nach den Nervenbahnen der Chromatophoren und ihre Bewegung, immerhin Aufklärung herrscht, so sind zur Beantwortung physiologischer Fragen spärlich eindeutige Ergebnisse vorhanden. Da die verschiedenen Autoren oft unter einander nicht vergleichbaren Versuchsbedingungen gearbeitet haben, so ist es schwierig, die Resultate für einen allgemeinen Gesichtspunkt zu verwerten. Dies gilt besonders für die Beobachtungen über Giftwirkung: nur wenige, einander meist widersprechende Angaben findet man hierüber. Die vorliegenden Untersuchungen gingen von dem Plan aus, auf diesem Gebiet zu einiger Klarheit zu gelangen, die Angaben zu ergänzen und, wo möglich, die Widersprüche aufzuklären.

Der enge Zusammenhang der Alkaloidwirkung mit dem Nervensystem, der diese Gifte gleichsam zu Analysatoren seiner Differenzierungen macht, brachte es mit sich, auch der Frage nach den Korrelationen zwischen Nervensystem und Farbenwechsel experimentell näherzutreten. 
Nur bei wenigen Fischarten gibt es einen so ausgesprochenen Farbwechsel wie bei den Pleuronectiden. Zwar haben wir hier keinen Farbenwechsel im eigentlichen Sinne, sondern nur einen Wechsel von hell und dunkel, der durch die Expansion und Kontraktion einer einzigen Art von Pigmentzellen, der schwarzen Chromatophoren (ïber die roten Chromatophoren siehe folgenden Abschnitt), bedingt ist, im Gegensatz zu anderen Fischen (und anderen Tieren), die durch verschiedenfarbige Chromatophoren - Xanthophoren, Phäophoren (Schmidt), Erythrophoren (Fuchs), Guanophoren (Schmidt) usw. - ausgedehnte Variationen in der Färbung erzielen. Die Chromatophorenreaktion tritt bei Pleuronectiden so prompt und intensiv ein, daß zu verwundern ist,

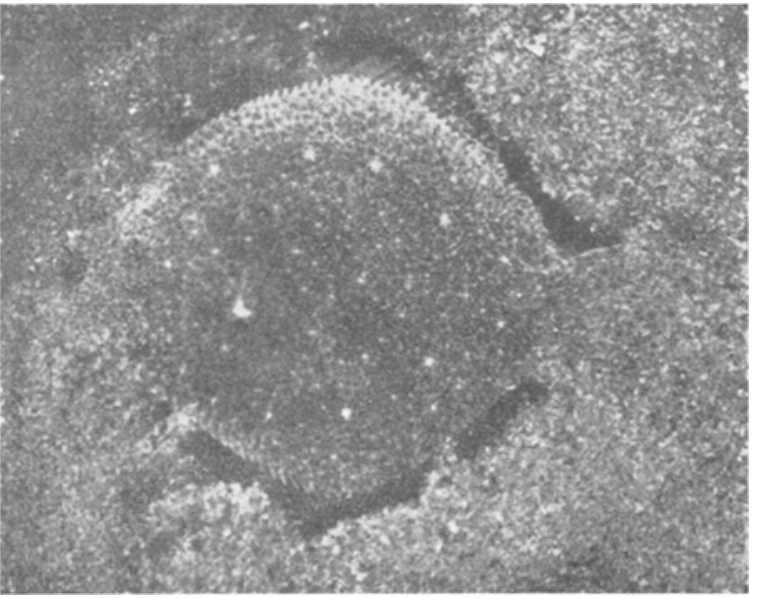

Abb. 1. Steinbutt auf Sandgrund, die Konturen vom Sand befreit. daß sehr wenige systematische Untersuchungen hierüber vorliegen. Die wichtigsten sind die von" Pouchet'), der den Grundstein zur Physiologie der chromatischeHautfunktion gelegt hat. An der Schnelligkeit des Farbwechsels wird wohl Rhombus maximus von keinem anderen Fisch übertroffen. Die Beobachtung der Tiere

im hiesigen Aquarium ergab ein Minimum von 3 Sekunden, welche genügten, um die hellgelbe Farbe, die Steinbutte beim ruhigen Liegen im Sande zeigen, in die dunkelgrau-braune zu verwandeln, die sie beim Umherschwimmen annehmen. Die Übereinstimmung mit dem Untergrund ist so täuschend, daß man auf den ersten Blick die Tiere von dem Sandboden, auf dem sie liegen, nicht unterscheiden kann. Die nebenstehende Reproduktion einer Photographie (aus der zoologischen Station Büsum), Abb. 1, gibt ein deutliches Bild von der Untergrundanpassung des Steinbutts (Rhombus maximus).

1) M. G. Pouchet, Du rôle des nerfs dans les changements de coloration des poissons. Journ. de l'anat. et de la physiol. norm. et pathol. de l'homme et des animaux. 8. 1872. - Sur les rapides changements de coloration provoqués expérimentalement chez les crustacés et sur les colorations bleues des poissons. Ibidem 8, 1872, und 9, 1873. - Des changements des colorations sous influence des nerfs. Ibid. 1876. 


\section{Bau der Chromatophoren von Pleuronectes platessa.}

Über die Lage der Chromatophoren in der Fischhaut sind wir durch die Untersuchungen von Cunningham ${ }^{1}$ ) orientiert. Sie liegen in der Cutis, zwischen Schuppenoberfläche und Epidermis. Thre Form ist bestimmt durch den jeweiligen physiologischen Zustand; bei der Expansion erscheinen sie sternförmig, mit radiär verlaufenden Lichtungen (Abb. 3 und 4). Abweichungen von dieser Form beobachtete Fran $z^{2}$ ) an Chromatopho-

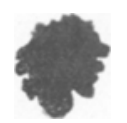

Abb. 2, Chromatophore yon Pleuronectes platessa, stark kontrahiert.

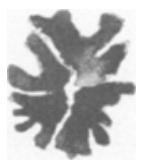

Abb. 3. Mäßig expandiert.

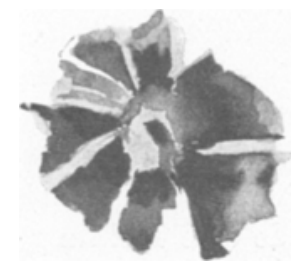

Abb. 4. Starke Expansion.

ren aus dem Flossensaum von Platessalarven, dessen Pigmentzellen langgestreckt sind. Übrigens sind diese Abweichungen sehr häufig, wie aus den verschiedenen Chromatophorentypen (Abb. 5, 6) ersichtlich ist.

Die Pleuronectiden haben relativ kleine Chromatophoren. Speziell bei Pleuronectes platessa sind zwei Arten zu unterscheiden. Die braunschwarzen, über die ganze Rückenseite verstreuten, und die gelb-roten, die immer in Gruppen zusammenliegen und die roten Flecken bilden. Beider Kontraktion erscheinen dieChromatophoren vollständig zu einer Kugel geballt, der Rand jedoch ist teil-

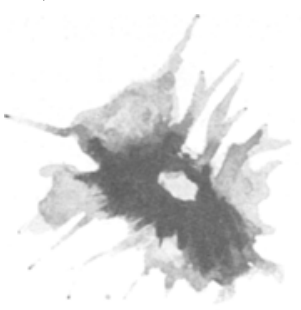

Abb. 5.

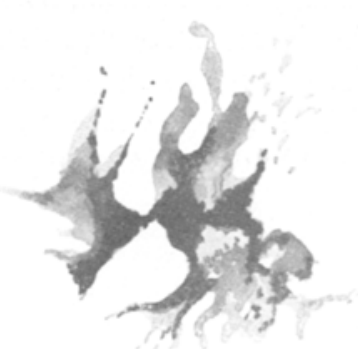

Abb. 6 . weise leicht gezackt. Die schwarzen Chromatophoren sind in der Rückenhaut ziemlich gleichmäßig verteilt.

Die dunkeln Flecken, die Zeichnung der Schollen, beruht also nicht auf einer größeren Dichte der Pigmentzellen an den betreffenden Stellen, sondern diese sind durch einen verhältnismäßig größeren Expansionszustand der Chromatophoren bedingt. Die roten Pigment-

1) J. Cunningham, An experiment concerning the absence of color from the lower side of flat-fishes. Zoolog. Anz. (I) 14. 1891.

2) V. Franz, Die Struktur der Pigmentzelle. Biol. Centralbl. 28, 536. 1908. 
zellen verharren dauernd in einem mittleren Expansionszustand. Eine Ballung tritt bei ihnen weder unter physiologischen Bedingungen noch auf elektrischen Reiz hin ein. Eine Aufhellung der roten Flecke wird lediglich dadurch erzielt, daß bei Reizung die zwischen ihnen liegenden schwarzen Chromatophoren sich kontrahieren.

Die Nervenendigungen in den: Chromatophoren von Pleuronectes platessa verlaufen nicht im Mittelpunkt der Zelle, sondern im peripherischen Protoplasma. Im Gegensatz zu anderen Fischchromatophoren sind die Nervenendigungen wenig verästelt [Ballowitz'1)]. Das Pigment ist schwarzbraun und fein granuliert. Dies ist jedoch nur an den Ausläufen der Chromatophoren und an Stellen, wo das Pigment teilweise zurückgezogen ist, sichtbar. Das Zentrum der Zelle ist, wenn nicht gerade eine Lichtung vorhanden ist, undurchsichtig schwarz. Einzelne Pigmentgranula liegen auch in der Umgebung der Chromatophoren. Soviel über die histologischen Tatsachen.

\section{Allgemeines über ihr Verhalten gegen Reize.}

Der Farbenwechsel und die Untergrundreaktionen sind bei Pleuronectes platessa nicht so evident wie z. B. bei Rhombus. Die Farbe ändert sich von hellgrau zu grauschwarz. Die Zeichnung bleibt beim Farbenwechsel unter normalen Umständen bestehen und ist immer dunkler als die übrige Haut. Nur bei einer Aufhellung durch Nachhirnreizung oder Adrenalin kann sie verschwinden. Vergleicht man die mikroskopischen Bilder von normalen Hautstücken mit solchen von dunkler Zeichnung, so sieht man, däß die Chromatophorenzahl für die gleiche Fläche bei letzteren nicht größer ist. Die Zeichnung kann also nur durch das Nervensystem bedingt sein und beruht auf einem größeren Tonus der Chromatophoren bestimmter Hautgebiete.

Daß das Licht für die Pigmentbildung von Wichtigkeit ist, geht daraus hervor, daß die bei der Wanderung der Augen zur Bauchseite gewordene Fläche ihr Pigment verliert. Experimentell kann durch dauernde Belichtung der weißen Unterseite diese wieder zur Pigmentbildung veranlaßt werden. Bei Blendung von Rhombus sah Pouchet2), daß den Tieren die Fähigkeit verloren ging, sich an den Untergrund anzupassèn, und daß sie eine mittlere Färbung annahmen. Bei Belichtung der Augen und bestimmter Hautbezirke mit einer starken Lichtquelle (Bogenlampe) konnte ich keine Änderung der Färbung feststellen.

Bei Beunruhigung verdunkelt sich Pleuronectes platessa stets. Diese Verdunklungsreaktion ist wohl auch als Ausdruck psychischer Erregung aufzufassen.

Bei mechanischer Reizung tritt eine Verdunklung an der Reizstelle auf.

1) E. Ballowitz, Zeitsehr. f. wissensehaftl. Zoologie 56. 1893.

2) Pouchet, 1.c. 
Nach elektrischer (faradischer) Reizung der Haut kontrahieren sich die Chromatophoren ziemlich schnell. Die Aufhellung beginnt an der Stelle, wo die Elektroden aufliegen und schreitet von da nach allen Richtungen fort. $\mathrm{Fuch}_{\mathrm{s}}{ }^{1}$ ) bemerkt $\mathrm{zu}$ den elektrischen Chromatophorenbeeinflussungen, daß man daraus keinen Schlu $B$ auf die direkte elektrische Reizbarkeit der Chromatophoren ziehen kann: „da eine Ausschaltung der Nerveneinflüsse nicht erfolgt ist, also alle Reizerfolge als indirekt durch Nervenreizung hervorgebracht gedeutet werden könnten". Ich versuchte, die Frage nach der direkten elektrischen Reizbarkeit der Chromatophoren an Pleuronectes zu entscheiden.

Schon die Verbreitung der Aufhellung läßt auf einen nervösen Vorgang schließen. Grenzt man ein Hautstück mit 4 Schnitten ab, so zeigt

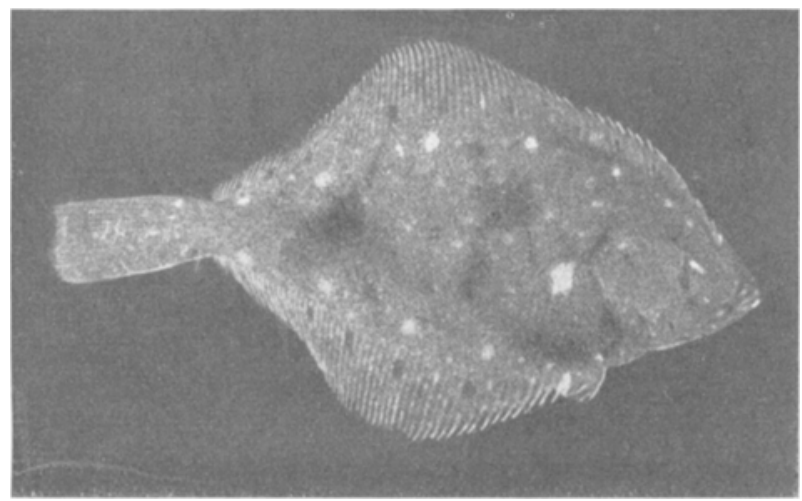

Abb. 7. Zeichnung und mittlere Hautfärbung von Pleuronectes platessa.

sich bei dessen faradischer Reizung, daß die Aufhellung des Hautvierecks an den Grenzen nicht halt macht, sondern ungestört weiter geht, was doch bei einer direkten Beeinflussung der Chromatophoren nicht zu erwarten gewesen wäre. Ferner spricht für die reflektorische Natur dieses Vorganges eine Beobachtung, die zuerst bei Versuch 12 gemacht wurde. Bei der Rückenmarksdurchschneidung wird der caudale Teil des Tieres dunkler. Hellt man nun den Teil zwischen zwei solchen Durchtrennungen faradisch auf, so gehen die Grenzen der Chromatophorenkontraktion durch die beiden Schnitte. Es entsteht ein helles Band, das dem Innervationsbezirk des betreffenden Abschnittes entspricht.

Da wir im Curare ein Mittel besitzen, die motorischen Nervenendigungen, also auch möglicherweise die pigmentomotorischen zu lähmen,

I) R. F. Fuchs, Der Farbenwechsel und die chromatische Hautfunktion der Tiere. Wintersteins Handbuch der vergleichenden Physiologie, Jena 1913. Bd. 3, I, 2. S. 1433 . 
wurde dieses Gift zur Entscheidung unserer Frage herangezogen. Bereits $\operatorname{Lode}^{1}$ ) hatte an curarevergifteten Forellen eine Ballung der Chromatophoren nach elektrischer Reizung beobachtet. Bei der curaresierten Scholle ließ sich nun ebenfalls eine Aufhellung nachweisen. Damit stände die direkte Reizbarkeit der Pigmentzellen durch den faradischen Strom a u Ber Z weifel. Jedoch besteht ein fundamentaler Unterschied zwischen der normalen und der Curareaufhellung; während die erstere schnell eintritt, sich über die ganze Haut verbreitet, beschränkt sich letztere auf die Reizstelle. Die Aufhellung geht von den Platinspitzen der Elektrode aus und erreicht allmählich einen Durchmesser von höchstens $3 \mathrm{~cm}$. Grenzt man jetzt ein kleines Hautstück mit Einschnitten ab, so geht die Aufhellung nicht weiter, sondern sistiert an den. Grenzen. Daraus folgt, daß es auf elektrischen Reiz hin eine direkte und eine reflektorische Chromatophorenreaktion gibt. Die gewöhnliche, auf einen elektrischen Reiz hin schnell auftretende, sich über größere Hautbezirke erstreckende Aufhellung ist reflektorischen Ursprungs.

Beim Absterben kontrahieren sich die Chromatophoren, es tritt eine postmortale Aufhellung ein, die bald einer maximalen Expansion der Chromatophoren weicht, die sich durch Reize nicht mehr rückgängig machen läßt. $\mathbf{F u c h s} \mathbf{s}^{2}$ ) hat darauf aufmerksam gemacht, daß

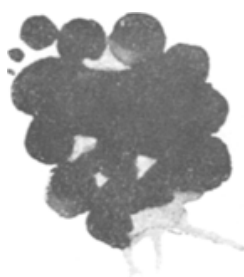

Abb. 8 . wir hierin einen der Totenstarre analogen Prozeß zu erblicken haben. Die postmortale Chromatophorenkontraktion ist ein weiterer Beleg für die Tatsache, daß alle lebendige Substanz im Kontraktionszustande abstirbt. ${ }^{3}$ ). (Degenerationskontraktion einer Chromatophore s. Abb. 8). Die Expansion der nun reaktionslos gewordenen Chromatophore entspräche der Lösung der Totenstarre. Ebenso wie die Totenstarre des. Muskels durch Temperaturerhöhung beschleunigt wird, so die post mortale Chromatophorenkontrak-

tion. Die durch Stoffwechselprodukte bedingte Zustandsänderung des Protoplasmas der Pigmentzelle führt deren Expansion herbei und ist analog der Lösung der Totenstarre. Man kann die Zustandsänderung als Gerinnungsvorgang durch postmortale Säurebildung auffassen. Die für die Totenstarre im allgemeinen geltenden Anschauungen. lassen sich auf diesen Fall übertragen.

1) Al. Lode, Beiträge zur Anatomie und Physiologie des Farbenwechsels der Fische. Sitzungsberichte der Kaiserl. Akad. d. Wiss. Wien, math.-naturwiss. Klasse 99, 3. 1890.

$\left.{ }^{2}\right)$ R. F. Fuchs, 1. c., S. 1428.

3) J. G. Schaefer, Die Totenstarre und ihre Beziehung zur Kontraktion. Biol. Centralbl. 40, 7, S. 316. 1920. 


\section{Beeinflussung der Chromatophoren durch das Nervensystem.}

Systematische Untersuchungen an Pleuronectiden über das Verhältnis des Zentralnervensystems zum Farbenwechsel liegen mit Ausnahme der Arbeiten von Pouchet ${ }^{\mathbb{1}}$ ) und Ba uer ${ }^{2}$ ) nicht vor. Den Einfluß des Gehirns untersuchte v. Frisch ${ }^{3}$ ) an Phoxinus laevis, Trigla und Salmo. Er fand, daß bei diesen Tieren in der Medulla oblongata ein Aufhellungszentrum gelegen ist. Bei dessen Ausschaltung trat eine sofortige Verdunklung ein. Reizung des Kleinhirns hatte keinen koloratorischen Erfolg. Mittelhirnreizung ergab kein eindeutiges Ergebnis. Im Zwischenhirn soll sich ein Hemmungszentrum befinden, welches das Kontraktionszentrum des Nachhirns hemmt. Dies schließt von Frisch aus der Verdunklung, die stets nach Zwischenhirnreizung eintrat.

Die Funktion des Rückenmarks als koloratorisches Organ suchte Pouchet (l. c.) an Rhombus klarzustellen. Da er bei caudaler Durchtrennung des Rückenmarks keinen Effekt erzieite, so schloß er, daß vom Gehirn zu den Chromatophoren keine Bahnen durch das Rückenmark gehen. Während diese Ergebnisse von Lode ${ }^{4}$ ) an Forellen und von van Rymberk ${ }^{5}$ ) an Solea bestätigt wurden, gelangten Le ydig ${ }^{6}$ ) und v. Frisch ${ }^{7}$ ) zu anderen Resultaten. Sie beobachteten bei Rückenmarksdurchtrennung eine Verdunklung. v. Frisch konnte durch Rückenmarkszerstörung die postmortale Aufhellung (Phoxinus 1.) verhindern, oder wenn sie bereits eingetreten war, rüekgängig machen.

Die Bedeutung des autonomen Nervensystems erwies zuerst Pouchet (1. c.) an Rhombus. Auf die Durchschneidung des Sympathicus im Hämalkanal folgte eine Verdunklung des caudal vom Schnitt gelegenen Teiles. Durchtrennung des N. trigeminus hat Verdunklung des Kopfes zur Folge. Die Versuche von v. Frisch ergaben, daß die Innervation der Chromatophoren von Phoxinus durch Vermittlung des Sympathicus geschieht.

1) M. G. Pouchet, Des changements des coloration sous influence des Nerfs. Journ. de l'Anat. et de la Physiol. 1876.

2) V. Bauer, Über die tonische Innervation der Pigmentzellen bei Plattfischen. Zentralbl. f. Physiol. 24, 724. 1910.

3) K. v. Frisch, Beiträge zur Physiologie der Pigmentzellen in der Fischhaut. Arch. f. d. ges. Physiol. 138, 319. 1911. - Utber farbige Anpassung bei Fischen. Zoolog. Jahrb. 32 (1912), Physiol. Abtl.

4) A. Lode, Beiträge zur Anatomie und Physiologie des Farbenwechsels der Fische. Sitzungsberichte der Kaiserl, Akad. d. Wiss. Wien, math.-naturwiss. Klasse. 99, Abtl. 3. 1890.

5) G. v. Rymberk, Ricerche sperimentali sulla metameria nel sistema nervoso simpatico I. La innervazione pigmento motorice. Arch. di fisiol. 1906.

6) Fr. Leydig, Integument und Hautsinnesorgane der Knochenfische Zoolog. Jahrb., Anat. Abtl. 8. 1895.

7) K. v. Frisch, l. c. 3). 
Daß diese Verhältnisse ohne weiteres auf Pleuronectiden übertragen werden dürfen, ist a priori anzunehmen. Jedenfalls erschien eine Nachprüfung wünschenswert. Durch die folgenden Experimente sollten Abweichungen festgestellt und vorhandene Tatsachen ergänzt werden.

Sämtliche Versuche sind, wenn nicht anders ausdrücklich vermerkt, an Pleuronectes platessa (Scholle) angestellt. Es wurden meist nur junge Tiere aus den Aquarien der zoologischen Station verwandt. Bei der Ausführung der Experimente unterstützte mich Herr med. Theodor Müller (Basel), dem ich auch an dieser Stelle hierfür meinen verbindlichsten Dank ausspreche.

\section{Der Einfluß des Gehirns auf den Farbenwechsel.}

Einem mittelgroßen Tier wurde das Gehirn bis zur Medulla oblongata freigelegt. Die Befestigung während der Operation wurde in einer mit Wachs ausgegossenen Blechwanne mit starken Nadeln bewerkstelligt. Der Untergrund war schwarz. Die Wanne wurde mit Seewasser gefüllt, bis die Scholle geràde bedeckt war.

Bei der Trepanation beginnt man am besten damit, den Schädel zwischen den Augen mit der Schere aufzuschneiden. Man sieht dann die beiden Optici und gelangt ohne Schwierigkeit zum Chiasma. Bei der Eröffnung der Schädelkapsel ist eine starke Blutung nicht zu vermeiden. Da das Blut sehr schnell gerinnt und das Operationsfeld unübersichtlich macht, so muß es mit einer Pipette sofort weggespritzt werden. Hat man den Knochen zurückgeklappt, so sieht man das Gehirn in Seitenansicht, da es unabhängig von der Lageänderung des einen Auges seine frühere Lage behalten hat. (Siehe Tafel.) Dann präpariert man vorsichtig ein kleines Stück der Medulla oblongata frei. Die Operation muß sehr schnell ausgeführt werden, da das Gehim infolge des ununterbrochenen Kreislaufes und des dadurch hervorgerufenen $\mathrm{O}_{2}$-Mangels leicht abstirbt.

Das Tier ist total dunkel. Die beiden Optici wurden faradisch gereizt, doch ohne Erfolg auf die Chromatophoren; ebenso erging es bei der Reizung des Groß- und Mittelhirns. Darauf wurden die Optici am Chiasma durchtrennt. Hierbei, wie bei Zwischenhirn- und Kleinhirnreizung, trat kein Farbenwechsel ein (nur eine heftige Innervation sämtlicher Muskeln) ${ }^{\mathbf{1}}$ ). Jedoch bei Nachhirnreizung (Medulla oblongata) kontrahierten sich die Chromatophoren. Die Aufhellung begann an der Schwanzflosse und ging langsam von da über das ganze Tier. Besonders intensiv war die Aufhellung jedoch nicht, was wohl mit dem durch die Dauer des Versuchs gesunkenen Erregbarkeitszustand des Gehirns zusammenhing. Nebenbei konnte noch eine herzhemmende Wirkung

1) Diese heftigen; wohl koordinierten Schwimmbewegungen bei Nachhirnreizung sprechen für die Annahme Steiners (Steiner, J., Die Funktionen des Zentralnervensystems und ihre Phylogenese, II. Abt.: Die Fische. Braunschweig 1888), daß auch bei den Fischen das ,,allgemeine Lokomotionszentrum“ des Körpers, dem die übrigen Bewregungszentren (des Rückenmarks) untergeordnet sind und dessen Zerstörung die Lokomotionsfähigkeit aufhebt, sich in der Medulla oblongata befindet. 
der Nachhirnreizung beobachtet werden (s. Abb.9u. 10), das Herz, das freigelegt war, setzte bei Reizung der Medulla oblongata für 10-20 Sek. aus.

Es war noch zu versuchen, ob sich am hellen Tier durch Hirnreizung keine Chromatophorenexpansion erzielen lasse, zu entschciden, ob das Gehirn hemmende Einflüsse bedingen kann.

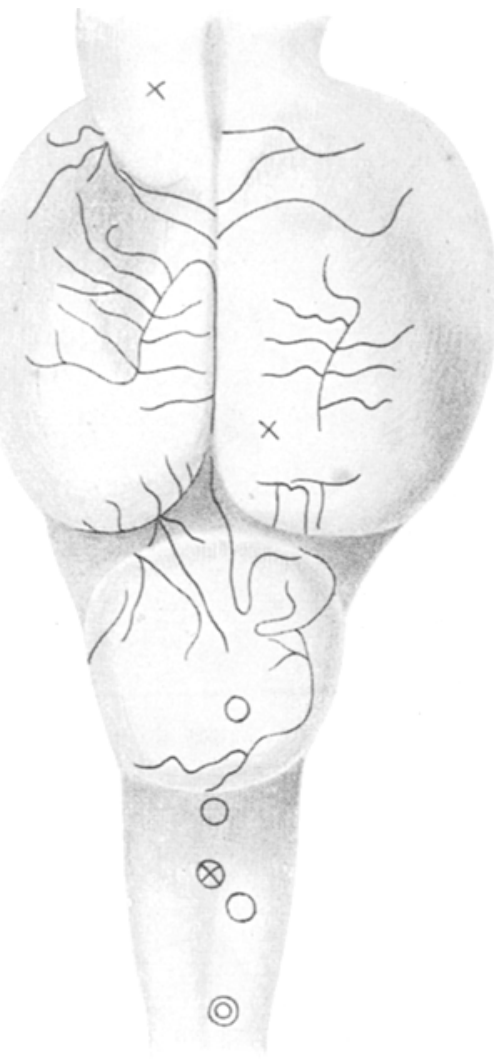

Abb. 9.

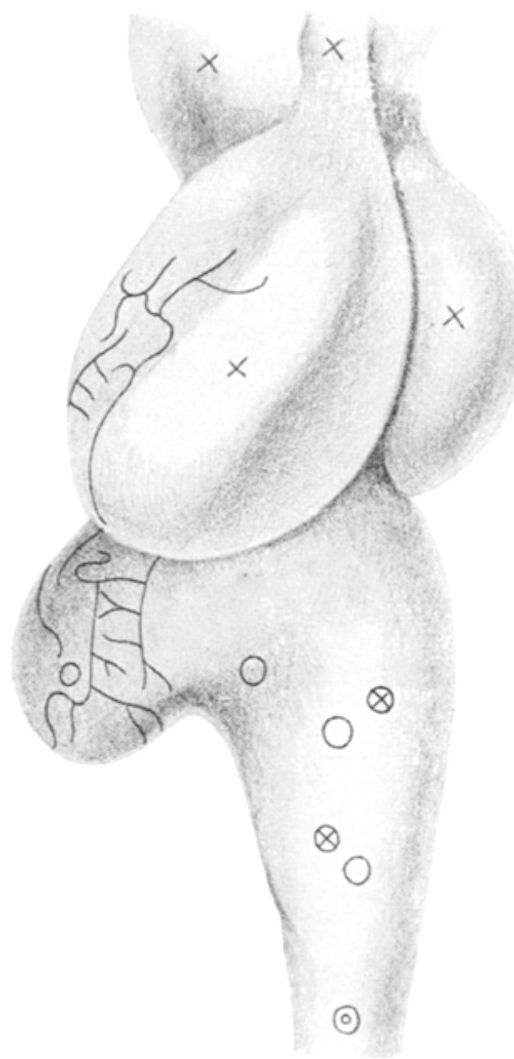

Abb. 10.

Abb. 9 und 10. Gehirn von Pleuronectes platessa. (Gefäße sind eingezeichnet.)

Stellen der Medulla oblongata, bei deren faradischer Reizung die intensivste Aufhellung eintritt.

() Stellen der Herzhemmung.

Stellen der motorischen Innervation (,allgemeines Lokomotionzentrum“ Steiners?). $\times$ Faradische Reizung ohne sichtbaren Frfolg.

18. (2. IX. 1920.)

Einer großen Scholle wurde auf hellgrünem Untergrund das Gehirn freigelegt. Das Tier war total dunkel. Durch Injektion einer minimalen Menge von Suprarenin wurde eine mittlere Färbung erreicht. Unter ganz besonderer Vorsicht wurde die Medulla oblongata präpariert. Es wurden nun, vom Opticus angefangen, alle Gehirnteile faradisch gereizt, jedoch ohne eine Verdunklung zu erzielen;

Pflügers Archiv f. d. ges. Physiol. Bd. 188. 
auch nicht bei $Z$ wischenhirnreizung. Jedoch trat beim Faradisieren des Nachhirns eine so intensive Aufhellung ein, wie noch nie beobachtet war. Setzte man die Elektrode auf bestimmte Bezirke der Medulla oblongata (s. Tafel), so waren in vier Sekunden die Chromatophoren der ganzen Haut so kontrahiert, daß das Tier weiß erschien. Sogar die sonst immer als dunkler Schatten sichtbare Zeichnung verschwand. Die Aufhellung überdauerte die Reizung. Wurde nur 2 Sekunden gereizt, so genügte dies, um die vollständige Aufhellung zu erzielen. Bei Zerstörung der Medulla oblongata trat eine maximale Verdunklung ein. Wie aus dem Versuch hervorgeht, konnten vom Gehirn aus die Chromatophoren nicht zur Expansion gebracht werden.

Allerdings könnte der Versuch aus dem Grunde nicht beweisend erscheinen, weil peripher vom Gehirn die sympathischen Nerven durch die Adrenalininjektion erregt waren und so eine zentrale Hemmung nicht zum Ausdruck kommen konnte. Aber wie aus der Verdunklung nach der Zerstörung des Zentrums im Nachhirn hervorgeht, war die Adrenalinwirkung schon abgeklungen. Auf jeden Fall zeigen die Experimente, daß auch bei Pleuronectes das Zentrum für die Kontraktion der Chromatophoren, das ,pigmentomotorische Zentrum", im Nachhirn liegt.

\section{Koloratorische Funktion des Rückenmarks und des autonomen}

\section{Nervensystems.}

Zur Untersuchung der Rückenmarksfunktion wurde die Durchschneidungsmethode angewandt. Im folgenden sollen einige Versuche beschrieben werden.

11. (30. VIII. 1920.) Das Rückenmark wurde zuerst bei I (Abb. 9) durchschnitten. Das Tier hatte normale Färbung. (Die Operation wurde auf schwarzem Untergrunde vorgenommen.) Jetzt wurde sofort der caudale Teil des Tieres

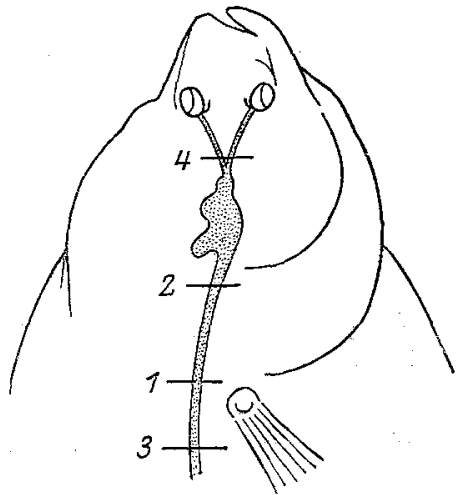

Abb. 11 dunkel. Durch den Schnitt schien also die Leitung des Chromatophorentonus unterbrochen zu sein. Der obere Teil einschließlich Kopf wurde heller, anscheinend durch Reizwirkung. Machte man nun bei 2 einen neuen Schnitt, so wurde die Zone zwischen 1 und 2 sowie der ganze Kopf maximal dunkel. Die Annahme eines Zentrums für die Chromatophorenkontraktion in der Medulla oblongata wird also bestätigt. Denn würde der Schnitt 2 etwas tiefer ausgefallen sein, so daß das Nachhirn intakt geblieben wäre, so hätte sich nur der Abschnitt 1 und 2 verdunkelt, nicht aber der übrige Teil, wie aus dem nächsten Versuch hervorgeht.

12. (30. VIII. 1920.) Das Rückenmark einer großen Scholle wurde von der Schwanzflosse aufwärts durchtrennt. (Färbung und Zeichnung normal, der Untergrund während der Operation schwarz.) Beim ersten Schnitt (Abb. 10) wurde die Schwanzflosse ein wenig dunkler. Beim zweiten Schnitt rückte die Verdunkelung bis dahin nach. Bei der dritten Durchschneidung war die Verdunkelung des unteren Teiles schon viel bedeutender. Die übrige Haut schien 
etwas heller geworden zu sein. Als das Mark bei 4 durchtrennt wurde, war zu beobachten, daß sich der Radius der Verdunkelungszone, je mehr die Schnitte nach oben gemacht wurden, vergrößerte. Dies ist anscheinend durch die Lage der peripherischen Nerven in den Innervationsbezirken der einzelnen Abschnitte bedingt. Bei jeder Durchtrennung war die Blutung sehr stark.

Man könnte einwenden, daß die Expansion der Chromatophoren durch die Unterbrechung der Blutzirkulation bedingt seị; aber v. Frisch ${ }^{\mathbf{1}}$ ) hat nachgewiesen, daß Anämie eine Aufhellung hervorruft, wahrscheinlich weil der $\mathrm{O}_{2}$-Mangel die Chromatophoren reizt. In unserem Fall tritt aber das Gegenteil ein.

Um zu entscheiden, ob die Ausschaltung des Rückenmarks die Verdunklung bedinge, wurde von Stelle 4 das Mark etwa $5 \mathrm{~cm}$ kopfwärts ausgebohrt $(A)$. Die V Verdunkelung hätte in dem ausgebohrten Teil

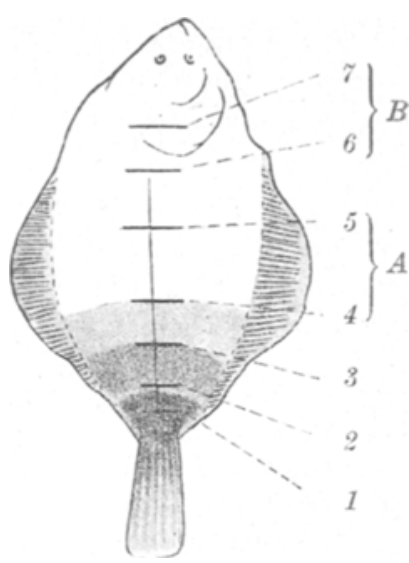

Abb. 12. nachrücken müssen. Aber die Aufhellung blieb dort bestehen. Erst als an der Stelle, bis wohin das Rückenmark zerstört war, ein neuer Schnitt angelegt wurde, wurde diese Zone dunkel. Dieser Versuch beweist, daß die koloratorischen Bahnen schon aus der Medulla ausgetreten sind, daB also der Chromatophorentonus bei Pleuronectes platessa durch den Sympathicus, der bei der Rückenmarksdurchschneidung gleichzeitig mit durchtrennt wurde, geleitet wird. Bei jedem folgenden Schnitt rückt die Verdunklung unter Wahrung scharfer Grenzen nach. Der obere Teil des Tieres ist sehr hell. Beim Freilegen des Gehirns wurde ein Nerv durchschnitten (Trigeminus?), worauf plötzlich eine maximale Verdunklung des Kopfes erfolgte. Es entstand eine ziemlich helle Zone zwischen Kopf und Schnitt 6. Als durch einen unglücklichen Zufall bei der Operation die Medulla oblongata zerriß, wurde dieser Abschnitt sofort dunkel. Darauf wurde der Wirbelkanal dieses Segments vom Schädel her ausgebohrt $(B)$. Würde der Chromatophorentonus vom Rückenmark hergeleitet, so hätte durch diesen Eingriff die Expansion der Chromatophoren noch vollständiger werden müssen. Aber das Gregenteil trat ein. Die Zone wurde wieder hell. Man könnte aus dieser Erscheinung schließen; daß im Rücken mark ein He m mungszentru m besteht, durch dessen Fortfall der Chromatophorentonus (der nunmehr nach Zerstörung des Nachhirns von den sympathischen Ganglien als sekundären Zentren ausgeht) wieder durchbricht. Auf jeden Fall geht deutlich aus diesen Versuchen hervor, daß das Rückenmark in direkter Beziehung für die Kontraktion der Chromatophoren nicht von

1) v. Frisch, l. e. 
integrierender Bedeutung ist, sondern daß sie dem a utonomen Nervensystem untersteht.

In jedem der durch die Schnitte entstandenen Segmente lassen sich die expandierten Chromatophoren durch faradische Reizung zur Kontraktion bringen. Jedoch bleibt die Aufhellung genau in den Grenzen des Innervationsbezirks des jeweiligen Rückenmarksabschnittes. Reizt man ein Segment, dessen Mark ausgebohrt ist $(A, B)$, so bleibt die eben erwähnte, als reflektorische Aufhellung zu charakterisierende Chromatophorenkontraktion aus, nur unmittelbar an den Elektroden sieht man eine Aufhellung (direkte Reizung). Das Rückenmark ist für das Zustandekommen der reflektorischen Aufhellung erforderlich.

19. (25. VIII. 1920.) Es wurde eine Hautstelle 10 Sekunden faradisch gereizt. Die Haut wurde in weitem Umkreise sehr hell. Dann wurde die Zeit bestimmt, welche nötig war, um die Chromatophoren nach der Reizung wieder zur Expansion zu bringen. Wurde nun das Rückenmark bei Beginn der Medulla oblongata durchschnitten, und man hellte eine Stelle faradisch 10 Sekunden auf, so sah man, $\mathrm{daß}$ die Chromatophorenkontraktion nach der Reizung sehr lange bestehen blieb, etwa um das 3-4fache wie vorher.

Auch dies läßt sich durch die Annahme erklären, daß vom Rückenmark hemmende Einflüsse zum pigmentomotorischen Zentrum im Nachhirn gehen.

\section{Giftwirkung und Farbenwechsel.}

In der Literatur sind nur vereinzelte Angaben über den Einfluß von Giften auf den Farbwechsel der Fische anzutreffen. Lode ${ }^{1}$ ) beobachtete an der Forelle nach subcutaner Injektion von Curare, das in Wasser und. Glycerin gelöst war, eine Verdunklung. Pouchet ${ }^{2}$ ) dagegen fand bei Curarevergiftung von Rhombus keine Anderung der Farbe. Morphin und Chinin hatten keine eindeutige Wirkung. Nach v. Frisch ${ }^{3}$ ) hat Chloralhydrat eine Expansion der Chromatophoren zur Folge. Eine 5 proz. Cocainlösung, lokal der Haut appliziert, bedingt an dieser Stelle Expansion. Dagegen trat nach Cocaininjektion eine Ballung der Chromatophoren ein. Wenn auch die Widersprüche bei den Beobachtungen nicht so verwirrend sind wie die über die Giftwirkung an Cephalopodenchromatophoren, so erreichen sie doch, ganz abgesehen von der großen Unvollständigkeit, nicht die Sicherheit der Ergebnisse an den Pigmentzellen der Amphibien, die wir den systematischen Untersuchungen von $\mathrm{Fuchs}^{4}$ ) zu verdanken haben.

Bei allen Farbwechselversuchen mit Giften fällt auf, daß die Chromatophoren selten in einer einzigen Weise reagieren. Meistens wird

1) Al. Lode, I. c. 1890.

2) M. Pouchet, l. c. 1872 and 1876.

3) v. Frisch, Arch. f. d. ges. Physiol. 138, 319. 1911.

4) R. F. Fuchs, Zur Physiologie der Pigmentzellen. Biol. Centralbl. 26. 1906. 
die Vergiftung mit einer Kontraktion der Chromatophoren, also Aufhellung, beantwortet. Darauf folgt dann eine Expansion der Pigmentzellen. So kommt es, daß die einen Autoren dies, die anderen jenes als die spezifische Giftwirkung betrachteten. Es ist in jedem Falle unbedingt eine Reizwirkung und eine (spezifische) Dauerwirkung zu unterscheiden. Unter Berücksichtigung dieser Tatsache wurden die folgenden Versuche angestellt.

$\mathrm{Zu}$ weiteren Beweisen für die Regulation des Farbenwechsels durch das autonome Nervensystem stehen zwei elektiv auf dieses wirkende chemische Substanzen zur Verfügung, das Nicotin, das die sympathischen Ganglien lähmt, und das Nebennierenextrakt Adrenalin, das diese Elemente erregt. Ich führe daher die Versuche über die Wirkung dieser Stoffe zunächst an.

9. (29. VIII, 1920.)

$$
\text { Nicotin. }
$$

I cem Nicotin auf $100 \mathrm{ccm}$ Aqua dest. Davon wurde $1 / 4 \mathrm{ccm}$ subcutan injiziert. Es trat eine cofortige sehr intensive Aufhellung ein, die auf die anfängliche Reizwirkung des Nicotins auf die sympathischen Ganglien zurückzuführen ist. (Intensives Excitationsstadium. Atmung sehr beschleunigt.) Dann wurde das Tier maximal dunkel.

Der Chromatophorentonus ist also durch die nun eingetretene Lähmung des Sympathicus a ufgehoben. Es war also zu erwarten, daß Adrenalin das Gegenteil bewirkt.

10. (29. VIII. 1920.)

\section{Adrenalin.}

$1 / 4$ ccm Solut. Suprarenini hydrochlor. synth. 1 : 1000 subcutan. (Untergrund schwarz.) Die Injektionsstelle wurde stark aufgehellt. Nach 30 Sekunden werden die dunklen Stellen der Hautzeichnung heller und hierauf die ganze Haut (lebhaftes Umhersohwimmen.) Die Zeichnung verchwindet ganz, die Hautfarbe ist weißgelb. Dann wurde das Rüickenmark im oberen Drittel durchschnitten. Die caudale Hälfte wurde maximal dunkel, während die helle Färbung des oberen Teiles bestehen blieb. Die Grenze war sehr scharf. Dieser Kontrast dauerte 10 Stunden.

Adrenalin erhöht also den Chromatophorentonus durch Erregung der sympathischen Nerven.

13. (31. VIII. 1920.)

$$
\text { Nicotin-Adrenalin. }
$$

Konzentration der verwandten Lösungen wie bei den Versuchen 9-10. (Untergrund dunkel.) Nach einer anfänglichen Erhellung (Reizwirkung) wird die ganze Haut nach der Nicotininjektion dunkel. Durchtrennung des Rückenmarks im caudalen Drittel ohne Einfluß. Ein zweiter Schnitt $1 \mathrm{~cm}$ höher kopfwärts ebenfalls ohne Erfolg. Darauf Adrenalin subcutan in der Gegend des Kopfes injiziert. Es trat nach $1 / 2$ Minute eine Aufhellung des Oberkörpers ein. Einige Stellen am Kopf (Kiemenrand) blieben jedoch dunkel, was wohl darauf zurïckzuführen ist, daß verschiedene Teile des sympathischen Nervensystems bereits abgestorben oder so stark durch das Nicotin gelähmt waren, daß die Lähmung sich durch Adrenalin nicht kompensieren ließ. Die koloratorischen nervösen 
Elemente des Kopfes scheinen also besonders empfindlich gegen Nicotinwirkung zu sein. Wurde das Rückenmark von der letzten Durchschneidung aus kopfwärts bis zur Medulla oblongata ausgebohrt, so wich diese Verdunkelung langsam einer Aufhellung.

Es ist also dern oberen Abschnitt des Rückenmarks ein hemmender EinfluB auf die Chromatophorenkontraktion zuzusprechen.

1. (16. VIIX. 1920.)

$$
\text { Morphin. }
$$

Es wurden $1 / 4 \mathrm{ccm}$ einer 1 proz. Lösung von Morphin. isovaler. subcutan injiziert. (Der Untergrund ist sehwarz.) An der Injektionsstelle trat in einer $1 / 2$ Winute eine lokale Verdunkelung auf, die sich durch faradischen Reiz nicht entfernen ließ. Die Färbung bleibt gleichmäßig dunkel.

14. (31. VIII. 1920.)

\section{Veratrin.}

$1 / 4$ ccm einer 0,5 proz. wässerigen Lösung von Veratrin. Zuerst trat eine Aufhellung ein, die auf die Reizwirkung zurïckzuführen ist. Die Injektionsstelle ist dunkel. Nach wenigen Minuten wurde die Färbung wieder normal. Darauf nochmals die gleiche Dosis, worauf das Tier dunkel wurde. Durchschneidung des Rückenmarks hat den gleichen Erfolg wie am normalen Tier.

3. (23: VIII. 1920.)

$$
\text { Atropin. }
$$

$1 / 2$ ccm einer 0,5 proz. Lösung von Atrop. sulf. injiziert. In 20 Sekunden ist eine totale Aufhellung des Tiers eingetreten. (Die Atmung ist sehr beschleunigt.) Diese Aufhellung ist auf eine Reizwirkung zurïckzuführen. Gleich darauf wurde die Haut gleichmäßig dunkel.

17. (31. VIII. 1920.)

$$
\text { Cocain. }
$$

$1 / 2$ cem 1 proz. Novocainlösung. Die Färbung bleibt unverändert. Die Injektionsstelle wurde dunkel. In 5 Minuten allgemeine Verdunkelung. Rückenmarksdurchschneidungen in den verschiedenen Lagen sind ohne Erfolg. Durch faradischen Reiz ist keine Aufhellung zu erzielen (mit Ausnahme der lokalen direkten Chromatophorenkontraktion). Ausbohren des Rückenmarkes bewirkt keine Aufhellung.

\section{8. (29. VIIT. 1920.)}

\section{Curare.}

$1 \mathrm{ccm}$ einer 1 proz. wässerigen Curarelösung. (Schwarzer Untergrund.) Injektionsstelle hell. In zwei Minuten Krämpfe und totale Verdunkelung. Reflexerregbarkeit erloschen. Rückenmarksdurchschneidung war ohne Einfluß. Die Curarewirkung ist also nicht zentral bedingt, sondern besteht in einer Läh. mung der pigmentomotorischen Nervenendigungen. Die Kontraktion der Chromatophoren durch faradischen Strom geht sehr langsam vor sich und beschränkt sich nur auf die Stelle, der die Elektroden aufliegen. Dies spricht dafür, dab-die normale Aufhellung durch faradischen Reiz reflektorischen Ursprungs ist. Wenn der Reflexweg durch die Curarewirkung unterbrochen ist, so reagieren die Chromatophoren doch auf elektrischen Reiz. Während die reflektorische Aufhellung fast augenblicklich nach Reizung auftritt und sich über große Hautabschnitte verbreitet, ist die letztere auf die Hautstelle beschränkt. 
Auf jeden Fall gibt es eine direkte Reizbarkeit der Chromatophoren, unter Ausschluß des Nervenweges.

5. (30. VIII. 1920.)

$$
\text { Strychnin. }
$$

$1 / 4 \mathrm{ccm}$ einer 0,1 proz. Lösung Strychnin nitr. (Untergrund dunkel.) In einer Minute ist das Tier aufgehellt (Reizwirkung). Die Injektionsstelle wird dunkel.

6. (30. VIII. 1920.)

$1 / 4$ ccm 0,1 proz. Strychn. nitr. Injektionsstelle dunkel. Allmählich wird eine geringe Aufhellung bemerkbar. (Stark beschleunigte Atmung.) Hierauf nochmalige Einspritzung von $1 / 4 \mathrm{ccm}$. Es traten die typischen Strychninsymptome auf und gleichzeitig eine starke Verdunkelung. Dann wurde das Rückenmark im oberen Teile $1 \mathrm{~cm}$ unter der Rückenflosse durchschnitten (Abb. 12 bei 3). Unmittelbar nach der Durchtrennung folgte eine totale Aufhellung oberhalb der Schnittstelle. Dann wurde das Rückenmark $1 \mathrm{~cm}$ höher (in der Abb. bei 1) kopfwärts durchschnitten, dieser Abschnitt' wurde ebenfalls dunkel. Die Grenze zwischen dem dunklen Teil und dem stark aufgehellten Kopf war ziemlich scharf. Die N. optici wurden durchschnitten (bei 4), ohne daß eine Änderung zu konstatieren gewesen wäre. Mit einem neuen Schnitt (bei 2) wurde die Medulla oblongata ausgeschaltet. Die helle Färbung des Kopfes wurde sofort dunkel.

15. (31. VIII. 1920.)

\section{Phenol.}

$1 / 2$ ccm 1 proz. Phenollösung. (Untergrund schwarz.) Sofort nach der Einspritzung setzt eine starke Chromatophorenkontralktion ein, die als Reizwirkung anzusprechen ist. Die Injektionsstelle bleibt dunkel. Darauf typische Phenolwirkung. Bej Eintritt der klonischen Krämpfe bleibt die Aufhellung bestehen. Bei Durchschneidung des Rückenmarks unten verdunkelt sich die Schwanzflosse. Die übrige Haut zeigt mittlere Färbung. Es wurde ein neuer Schnitt durch die Mitte des Rückenmarks gemacht und von da aus das Mark bis zur Mitte der Medulla oblongata ausgebohrt. Hierauf kontrahieren sich die Chromatophoren des Kopfes maximal (die unteren sind infolge der Sympathicusdurchschneidung reaktionslos geworden).

Da bei fast allen Experimenten an der Injektionsstelle eine lokale Chromatophorenexpansion beobachtet wurde, so sollten durch die folgenden Versuche die Bedingungen für diese Erscheinung klargelegt werden.

Zunächst war es möglich, daß die Verdunklung durch das Einstechen der Kanüle als mechanischer Reiz verursacht wurde. Es zeigte sich aber, daß das bloße Einstechen ohne Einwirkung war. Weiter konnte die Verdunklung durch die osmotische Reizwirkung der injizierten Lösungen bedingt sein. Jedoch trat sowohl nach Einspritzung von destilliertem Wasser wie einer Salzlösung keine Chromatophorenreaktion an der Injektionsstelle auf. Also ist die bei der Injektionsstelle aller Alkaloide auftretende lokale Verdunklung a uf einen spezifisch lähmenden Einfluß dieser Gifte sowohl auf die Chromatophoren direkt als auch auf ihre afferenten Nerven (wofür die scharfe Begrenzung und die gleichmäßige Expansion aller Chromatophoren dieser betreffenden Hautstelle sprechen) zurückzuführen. 


\section{Giftwirkung auf isolierte Hautstellen.}

Es wurden einzelne Hautstücke, sowohl helle wie dunkle, excidiert und in folgenden Lösungen untersucht:

Cocain lähmt an herausgeschnittenen Hautstücken die Chromatophoren. Die Ballung der Pigmentzellen weicht einer Expansion.

Adrenalin wirkt a uch lokal. Dunkle Hautstellen werden sofort nach Einbringen in Adrenalinlösung hell.

Strychnin und Nicotin sind ohne Einfluß.

Die Interpretation der Ergebnisse ist naturgemäß schwierig, da die Giftwirkung nie nur eine einzige, spezifische ist, sondern manche Nebenwirkungen hat. Bei verschiedenen Dosierungen treten dann die verschiedenen Nebenwirkungen mehr in den Vordergrund. Dies ist der Grund der sich widersprechenden Behauptungen, die bei den Untersuchungen über die Giftwirkungen zu finden sind.

Der Angriffspunkt eines Giftes ist wohl nach der Eigenart dieser Substanz ein spezifischer (z. B. die sensiblen Hinterhornganglien für Strychnin, die motorischen Vorderhornganglien für Phenol). Aber dies ist nicht der einzige (z. B. curareähnliche Wirkung des Strychnins bei größeren Dosen, Sympathicuserregung usw.). Alle diese anderen Einflüsse können bestimmend auf die zu untersuchende Lebenserscheinung einwirken, mit der typischen Giftwirkung interferieren und diese aufheben, so daß es also nicht gerechtfertigt ist, den beobachteten Erfolg als spezifische Wirkung aufzufassen. Diese Faktoren sollen im folgenden Abschnitt berücksichtigt werden.

\section{Analyse der Giftwirkungen.}

Bewirkt irgendein Alkaloid z. B. eine Expansion der Chromatophoren, so ist es ohne weiteres noch nicht entschieden, welche Teile des Nervensystems beeinflußt worden sind, ob das Zentrum, Sympathicus oder die peripheren Nerven gelähmt sind. Dies ist nur der Fall bei Giften von solch elektiver Wirkung wie Curare, Nicotin (u. a. m.). Aber bei allen anderen müßte ein experimenteller Nachweis über den Angriffsort erbracht werden. In den oben erwähnten Versuchen ist dies nur teilweise geschehen. Um nun doch zu einer Vorstellung hierüber zu kommen, ist es vielleicht zweckmäßig, ein Vergleichsobjekt heranzuziehen, über das in toxikologischer Beziehung Näheres bekannt ist. Es müßte ein dem Chromatophorensystem ähnliches System sein, also vom Sympathicus dirigiert werden, untex einem Tonus stehen, ${ }^{2}$ dessen Zentrum an der Medulla oblongata liegt usw. Beide Systeme müßten 
analoge Funktion besitzen. Über die Funktion der Chromatophoren hat sich $\mathrm{Fuchs}^{\mathbf{1}}$ ) zuerst klar ausgesprochen.

Tiere, die einen durch Chromatophoren bedingten Farbenwechsel haben, sind poikilotherme Tiere, die also ihre Körpertemperatur mit der des Mediums ändern. Sie haben im Gegensatz zu den homoiothermén Tieren nur eine physikalische, nicht aber auch eine chemische Temperaturregulierung ${ }^{2}$ ). Um nun einigermaßen die Temperaturschwankungen zu kompensieren, so verändern die Chromatophoren je nach ihrem Ballungszustande die Wärmestrahlungsabsorption und Wärmeleitung. Die chemische Wärmeregulierung wird also ersetzt dureh die Chromatophoren als physikalische Wärmeregulatoren. Die analoge Funktion beim Warmblüter hat das Gefäßsystem. Das Blut ist das bedeutendste Transportmittel der Wärme im Körper. Da die Wärmevermittlung auf der Oberfläche stattfindet, sind die Hauptgefäße regulatorisch von größter Bedeutung ${ }^{3}$ ).

Wenn beide, das Chromatophoren- und Gefäßsystem, in Innervation und Funktion übereinstimmen, so lassen sich noch weitere Analogien im speziellen nachweisen. Die Gefäßnerven treten mit den Kolorationsnerven aus dem Rückenmark in den Grenzstrang des Sympathicus über und gehen von dem Grenzstrang auf dem Wege spinaler und cerebraler Nerven zu ihrem Innervationsgebiet. Sowohl Gefäß- wie Chromatophorenzentrum sind sehr empfindlich gegen $\mathrm{O}_{2}$-Mangel. Ebenso wie sich einige Zeit nach Zerstörung der vasomotorischen Zentren in der Medulla ein never Tonus einstellt, dessen Zentrum die sympathischen Ganglien geworden sind, so ist nach $\mathrm{Fuchs}^{4}$ ) auch beim Chromatophorensystem die Möglichkeit eines sekundären bzw. tertiären Zentrums in den Ganglien des Sympathicus vorhanden.

Wir sind also berechtigt anzunehmen, daß die Feststellungen über die Giftwirkungen auf das Gefäßsystem auch für das Chromatophorensystem Geltung haben.

Nicotin: Zuerst intensive Aufhellung, dann maximale Verd unklung. Größere Mengen von Nicotin haben curareartige Wirkung auf die Nervenendigungen. Daß diese aber nicht die Chromatophorenexpansion bedingt, geht daraus hervor, daß die Nicotinwirkung durch Adrenalin kompensiert werden kann. Die anfängliche Aufhellung kann zustande kommen dadurch, daß das pigmentomotorische Zentrum in der Medulla oblongata gereizt wird, oder daß die sympathischen Ganglienzellen vor ihrer Lähmung stark gereizt werden. Beide Fälle

1) R. F. Fuchs, Die physiologische Funktion des Chromatophorensystems als Organ der physikalischen Wärmeregulierung der Poikilothermen. Sitzungsberichte der Physik.-med. Societät in Erlangen 44. 1912.

$\left.{ }^{2}\right)$ M. Rubner, Die Gesetze des Energieverbrauches bej der Ernährung. Leipzig-Wien 1902.

3) Auf den Einwand V. Bauers (Zeitschr. f. allg. Physiol. 16, 1914), daß diese Theorie nur auf einige Amphibien und Reptilien anwendbar sei, nicht aber auf die im Wasser lebenden Krebse und Fische, kann hier nicht eingegangen werden.

4) R. F. Fuchs, Wintersteins Handbuch, 1. c. S. 1439. 
sind bei der Nicotinwirkung auf das Gefäßsystem verwirklicht. Das vasomotorische Zentrum wird erregt und die peripheren Ganglienzellen. Beides bedingt eine Blutdrucksteigerung, darauf folgt, analog der Chromatophorenexpansion, eine Erschlaffung der Gefäße.

Adrenalin: Intensive Aufhellung. Durch Erregung der sympathischen Nerven erfolgt eine Kontraktion der Chromatophoren. Die Wirkung auf das GefäBsystem ist analog. Da es ein Produkt innerer Sekretion ist, was den Gefäßtonus beeinflußt, so ist die Annahme, daß der Chromatophorentonus ebenso vielleicht durch dasselbe reguliert wird, sehr wahrscheinlich, besonders da man bei Fischen Drüsen mit innerer Sekretion gefunden hat; deren Funktion noch einer Erklärung harrt. (Pleh n, Anatom. Anz. 28 1906, 197.) Fuchs ${ }^{1}$ ) betont die Möglichkeit, daß der Chromatophorentonus außer durch thermische Reize durch Stoffwechselprodukte bedingt sein könnte. Die Nicotinverdunklung wird durch Adrenalin aufgehoben. Zwischen beiden besteht ein Antagonismus, der sich ebenfalls dadurch kundgibt, daß die Nicotineinfuhr in den Warmblüterkörper die Adrenalinsekretion vermehrt ${ }^{2}$ ).

Morphin: Verdunklung. Diese ist nicht auf Sympathicuslähmung zurückzuführen. Nach Cl. Bernard ${ }^{3}$ ) wird dieser durch Morphin in normalen Dosen nicht gelähmt. Die Verdunklung ist bedingt durch die Lähmung des pigmentomotorischen Zentrums in der Medulla oblongata. Auch die übrigen Zentren des Nachhirns werden geschädigt. Analog dem Chromatophorensystem nimmt auch der Tonus des Gefäßsystems ab. Der Blutdruck sinkt enorm [Barbour $\left.\left.{ }^{4}\right)\right]$. Da auch die peripheren Nerven unbeeinflußt bleiben, so ist die Chromatophorenerschlaffung zentral bedingt. Es wäre zu untersuchen, wie die Nachhirnreizung und Adrenalinwirkung ausfiele. Letztere müßte, da sie an peripher gelegener Stelle wirkt, erfolgreich sein.

Veratrin: Zuerst Aufhellung, dann mäBige Verdunkl ung. Beides zentral bedingt. Das Chromatophorenzentrum wird zuerst erregt und dann gelähmt. Analog ist beim Gefäßnervensystem nach Veratrin zuerst Erregung (Kontraktion der Gefäße), darauf Lähmung (Expansion) des vasomotorischen Zentrums zu beobachten. Die Blutdrucksenkung nach Veratrin ist aber nicht sehr ausgesprochen, ebensowenig wie die Expansion der Chromatophoren.

Atropin: Totale Aufhellung, darauf Verdunklung. Die Chromatophorenkontraktion scheint zentral bedingt zu sein. Auch am

1) R. F. Fuchs, Wintersteins Handbuch, I. c. S. 1443.

2) Dale und Laidlaw, Journ. of physiol. 44. Proceed. 12.

3) Cl. Bernard, Über die Rolle der lähmenden Reflexwirkungen bej dem Sekretionsphänomen. Journ. de l'Anat. et Physiol. 64, 507.

4) H. Barbour, Zwei Typen der periodischen, durch Morphin hervorgerufenen Atmung. Journ. of pharmacol. a. exp. therapéut. 5393. 
Gefäßnervensystem ist eine Erregung des vasomotorischen Zentrums zu beobachten. $\mathrm{Ob}$ die darauffoigende Chromatophorenexpansion auf zentraler oder peripherer Lähmung beruht, ist nicht mit Sicherheit zu entscheiden. Der Sympathicus mit seinen Ganglien ist sehr resistent gegen Atropin ${ }^{1}$ ). Nur relativ große Dosen schädigen ihn. Es bleibt also die Möglichkeit einer zentralen Lähmung oder einer Ausschaltung der Nervenendigungen in den Chromatophoren bestehen. Eine Entscheidung könnte durch Nachhirnreizung oder Adrenalin herbeigeführt werden. Tritt eine Aufhellung ein, so ist der letztgenannte Fall ausgeschlossen.

Cocain: Verdunklung. Cocain erregt anfänglich die Zentren der Medulla oblongata. Daß aber keine Aufhellung erfolgt, liegt daran, daß periphere Elemente gelähmt werden und so die Chromatophorenkontraktion nicht zustande kommen lassen. Da von einer sympathicuslähmenden Wirkung des Cocains nichts bekannt ist, so bleibt nur die Annahme einer motorischen Lähmung übrig. Beweisend für die Annahme wäre die Tatsache, daß Nachhirnreizung und Sympathicusreizung erfolglos blieben.

Curare: Totale Verdunklung. Die schädigende Wirkung von Curare auf das Zentralnervensystem ist gering. Die Lähmung ist peripherisch. Die pigmentomotorischen Nervenendigungen werden ebenso wie die übrigen motorischen durch Curare geläh mt, was eine Expansion aller Chromatophoren zur Folge hat. Das Gefäßsystem reagiert ganz analog wie das Chromatophorensystem auf Curare. Auf jede Curareinjektion folgt eine Blutdrucksenkung, die unabhängig vom Herzen und von den Herznerven ist. Tillie ${ }^{2}$ ) findet eine vollkommene Analogie zur Lähmung der peripheren Nerven in der Lähmung der Gefäßnerven durch Curare.

Strychnin: Geringe Aufhellung, darauf totale Verdunklung. Strychnin reizt die Zentren der Medulla oblongata. Daher resultiert die Aufhellung. Die Sympathicuserregbarkeit wird gesteigert ${ }^{3}$ ). $\mathrm{Zu}$ entscheiden wäre, ob die Chromatophorenexpansion auf einer lähmung des Zentrums beruht oder durch die curareähnliche Wirkung des Strychnins auf die motorischen Nerven bedingt ist. Da aber nach Rückenmarkdurchtrennung wieder eine Aufhellung eintritt, so sind diese Möglichkeiten ausgeschlossen. Es bleibt also nur die Annahme übrig, daß durch Strychnin der hemmende Einfluß des Rückenmarks vergrößert wird.

$\left.{ }^{1}\right)$ J. Langley, Über die Physiologie der Speichelsekretion. Journ. of physiol. 1, 9, 10, 11.

2) J. Tillie, Über die Wirkung des Curare und seiner Alkaloide. Arch. f. experim. Pathol. u. Pharmakol. 2r.

$\left.{ }^{3}\right)$ M. Schiff, Bericht über einige Versuchsreihen. Arch. f. d. ges. Physiol. 4. 
Pheriol: Starke A ufhellung - mittlere Färbung. Von einer zentralen Erregung stammende Chromatophorenkontraktion. Die Rückenmarkserregbarkeitssteigerung hatte scheinbar eine Hemmung zur Folge.

\section{Zusammenfassung und Schluß.}

Bei elektrischer Reizung der Fischhaut läßt sich die Reaktion der Chromatophoren in zwei verschiedene Vorgänge scheiden:

I. Es erfolgt eine reflektorische Chromatophorenkontraktion. Daß diese Reaktion a uf dem Wegè der Nervenleitung vor sich geht und nicht a uf direkter Beeinflussung beruht, wird bewiesen durch folgende Tatsachen:

1. Die Aufhellung geht von der Reizstelle gleichmäßig über den ganzen Körper, und zwar gleichmäßig ohne Dekrement in der Intensität der Aufhellung.

2. Wird der Zusammenhang der Haut der Reizstelle mit der der Umgebung unterbrochen und nur mit dem Muskel in Verbindung gelassen, so geht die Aufhellung doch über die operativ abgegrenzte Hautstelle hinaus und erreicht unbekümmert ihre normale Ausdehnung, obschon der elektrische Reiz unbedingt lokal beschränkt ist.

3. Wird das Rückenmark samt dem Sympathicus in verschiedenen Höhen durchtrennt und einer der nun dunkel gewordenen Abschnitte faradisch géreizt, so springt die Aufhellung nicht auf ein anderes Segment über, sondern bleibt an den 'Innervationsbezirk des jeweiligen Rückenmarksabschnittes gebunden.

II. Es gibt eine direkte Chromatophorenbeeinflussung. Die Chromatophoren kontrahieren sich ohne Vermittlung des Nervensystems direkt.

1. Nach Curarevergiftung, durch die also alle Nerveneinflüsse ausgeschaltet werden, kontrahieren sich die Chromatophoren, jedoch nur lokal und begrenzt an der Elektrodenstelle.

2. Isoliert man an der curaresierten Scholle ein Hautstück von der Umgebung, so bleibt bei Reizung die Aufhellung auf die umgrenzte Stelle beschränkt, da die Reizwirkung auf curaresierte Chromatophoren nur lokal sein kann.

Der Aufhellungsreflex und die direkte Aufhellung unterscheiden sich durch Intensität, Schnelligkeit und Umfang der Aufhellung. Belichtung der Augen und der Haut waren ohne Einfluß auf den Farbenwechsel.

Aus den Durchschneidungs- und Reizversuchen am zentralen und autonomen Nervensystem folgt:

Durch Reizung sämtlicher Gehirnteile und des Opticus ließ sich keine Kontraktion der Chromatophoren erzielen. Ebenso Jieß sich von dort aus keine Hemmung nachweisen.

Bei elektrischer Reizung der Medulla oblongata wurde 
eine sofortige sehr intensive Aufhellung erzielt. Das pigmentomotorische Zentrum liegt also im Nachhirn. Zerstörung der Medulla oblongata hat eine sofortige maximale Expansion der Chromatophoren zur Folge.

Die bei Rücken marksd urchschneidung eintretende Verdunklung des caudalen Teils kommt dadurch zustande, daß hierbei gleichzeitig der $S y$ mpathic us mitd urchschnitten wird. Zerstörung des Rücken marks hat keinen Einfluß auf die geballten Chromatophoren. Ausbohrung ist also ohne Erfolg; erst durch die mit der Durchschneidung verbundene Sympathicuszerstör ung wird die Expansiondad urch herbeigeführt, da $B$ die den Tonus vermittelnde Bahn unterbrochen ist. Bei der Durchschneidung des Rückenmarks (und Sympathicus) wird der caudale Teil dúnkel, der obere aber heller als ursprünglich. Ob dies a uf Vermittlung von erregenden oder auf Unterbrechung von hemmenden Einflüssen be. ruht, ist unentschieden.

Daß das Rückenmark oder wenigstens der obere Teil hemmend wirken kann, geht aus folgendem hervor:

1. (12.) Durch Zerstörung des Zentrums und der Medulla oblongata maximale Expansion. Als das Rückenmark vom Schädel aus zerstört wurde, trat sofort Aufhellung ein.

2. (15.) An einer pheno'vergifteten Scholle wurde das Rückenmark von der Mitte bis zur Medulla oblongata ausgebohrt. Da in der Mitte Rückenmark und Sympathicus durchschnitten waren, so war der caudale Teil des Tieres dunkel, nach der Ausbohrung wich die mittlere Färbung einer intensiven Aufhellung.

3. (19.) Die elektrische Aufhellung geht in ganz kurzer Zeit nach Aufhören des Reizes zurück und weicht der ursprünglichen dunklen Hautfärbung. Hellt man nun eine Hautstelle auf und schneidet sofort das Rückenmark bei Beginn der Medulla oblongata durch, so bleibt die Aufhellung länger bestehen.

4. (13.) Die dunkle Färbung des Kopfes einer nicotinvergifteten Scholle wird nach Rückenmarkszerstörung aufgehoben.

5. (5.) Durchschneidet man an der strychninvergifteten Scholle das Rückenmark, so kontrahieren die vorher maximal expandierten Chromatophoren sich im oberen Teil.

Chemische Substanzen, die von spezifischer Wirkung auf den Sympathicus sind, haben einen eminenten Einfluß auf die Färbung.

Nicotin, dasdies ympathischenGanglien lähmt, bewirktExpansion. Adrenalin, das Elementedessympathischen Nervensystems erregt, bewirkt Kontraktion der Chromatophoren. 
Die Wirkung der übrigen Alkaloide ist aus folgender Tabelle ersichtlich:

Tabelle. Wirkung der Gifte auf die verschiedenen Elemente des Chromatophorennervensystems.

\begin{tabular}{|c|c|c|c|c|c|c|c|c|c|c|}
\hline \multicolumn{2}{|c|}{$\begin{array}{l}\text { Ghromatophoren- } \\
\text { Nervensystem }\end{array}$} & $\begin{array}{c}\text { Nico- } \\
\text { tin }\end{array}$ & $\begin{array}{l}\text { Adre- } \\
\text { nalin }\end{array}$ & $\begin{array}{l}\text { Mor- } \\
\text { phin }\end{array}$ & $\begin{array}{l}\text { Vera- } \\
\text { trin }\end{array}$ & $\begin{array}{l}\text { Atro- } \\
\text { pin }\end{array}$ & Cocain & Curare & $\begin{array}{c}\text { Strych- } \\
\operatorname{nin}\end{array}$ & Phenol \\
\hline \multirow{3}{*}{ 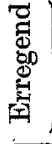 } & peripheres & & & & & & & & & \\
\hline & autonomes & $x$ & $\times 0$ & & & & & & $x$ & \\
\hline & zentrales & . & & & $x$ & $x$ & & & $x O)_{*}$ & $\times O)_{*}$ \\
\hline \multirow{3}{*}{ 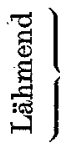 } & peripheres & & & & & $0 ?$ & 0 & 0 & & \\
\hline & autonomes & 0 & & & & $\therefore$ & & & & \\
\hline & zentrales & & & 0 & 0 & 0 & & & & \\
\hline
\end{tabular}

\section{Weitere Untersuchungen.}

Zunächst wurden Versuche mit Raja clavata angestellt. Die. Tiere wurden einmal in einem Gefäß mit schwarzem, darauf in einem mit weißem Untergrund gehalten. Selbst bei längerem Aufenthalt war keine An passung an den Untergrund zu erkennen. Elektrische Hautreizung, selbst von faradischen Strömen, blieb auf die Färbung ohne Einfluß.

Nebenbei wurde an Raja clavata eine interessante Erscheinung beobachtet, die ich an dieser Stelle erwähnen will. Ergreift man ein Tier schnell, legt es auf den Rücken und hält es einen Augenblick fest, so sieht man nach Aufhören der spontanen Bewegungen, daß das Tier regungslos mit ventral umgebogenen Seitenflossen liegen bleibt (Abb. 11). In dieser sonderbaren Lage bleibt das Tier liegen, bis die spontanen Bewegungen einsetzen, 'um die normale Körperlage wieder zu erlangen; auch durch künstlichen Reiz kann man beim flach auf dem Rücken liegenden Rochen diese Stellung erzielen (durch Auflegen der Elektroden unterhalb des Unterkiefers, M. coraco-mandibularis). Wir haben hier einen Fall von Reflextonus, wie er den früher als hypnoseähnlichen Erscheinungen aufgefaßten Zwangsstellungen verschiedener Tiere zugrunde liegt (Krebs, Frosch, Huhn, ägypt. Brillenschlange, Meerschweinchen [Verworn], Küchenschabe [Sozymansky]). Das Wesentliche dieser Erscheinung beruht nach Verworn ${ }^{1}$ ) im tonischen Lagereflex, der die sonderbare Haltung der Tiere bedingt. Naoh einer Analyse ist der Vorgang so zu denken, daB bei einer anormalen Lage des Tieres das cerebrale Lagereflexgebiet erregt wird, dagegen die motorischen Sphären des Großhirns gehemmt werden. Der Lagekorrektionsreflex kommt nicht zur Ausführung; durch den andauernd einwirkenden Reiz der abnormen Körperlage geraten die Zellen des Lagereflexgebietes in eine tonische Dauererregung. Die unter sonstigen Umständen am Lagekorrektionsreflex beteiligten Muskeln befinden sich in tonischer Contractur. Das Großhirn wirkt am Zustandekommen dieses Vorganges nur durch Hemmung der motorischen Rindengebiete mit. Die spon-

1) M. Verworn, Die sogenannte Fypnose der Tiere. Zentralbl. f. Physiol. 12 1898. 500 . 
tanen Bewegungen des Tieres zur Befreiung aus seiner Lage werden ausgeschaltet. Die tonische Contractur der Bauch- und Flossenmuskeln des Rochens ist der Ausdruck des am Erfolg verhinderten Umdrehreflexes.

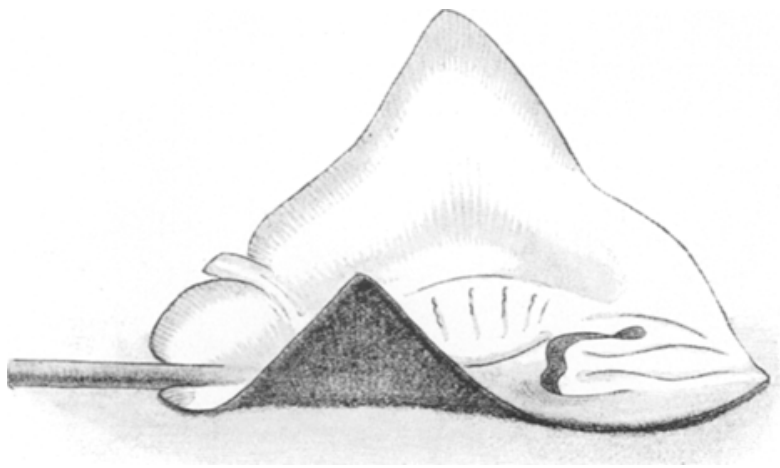

Abb. 13.

Am Glattrochen (Raja batis) war ebenfalls kein Farbwechsel zu beobachten.

Ferner wurden verschiedene Versuche mit Cyclopterus lumpus (Lumpfisch) angestellt.

Es waren kleine Tiere von durchschnittlich $10 \mathrm{~cm}$ Länge. Thre normale Färbung ist violettblau mit dunkler Längsstreifung.
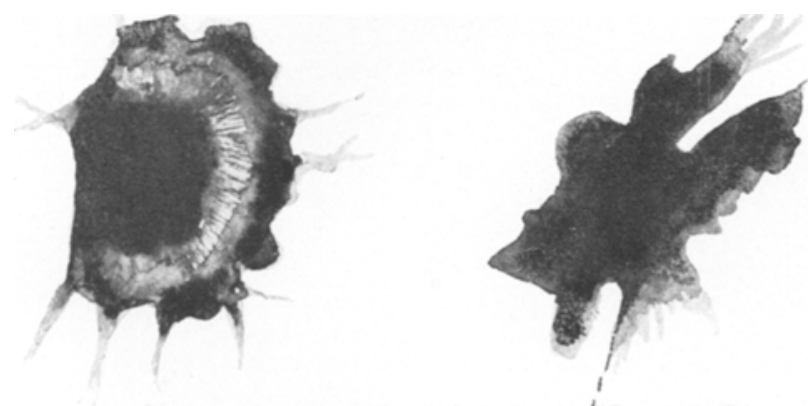

Abb. 14 u. 15. Chromatophoren von Cyclopterus lumpus (tiefe Hautschicht).

Die schwarzen Chromatophoren sind auf zwei Hautschichten verteilt, während die farbigen fast nur in der oberen Schicht liegen. Die Größe der schwarzen Chromatophoren in beiden Schichten ist nicht gleich, die unteren sind etwas größer. Besonders große liegen dort in der Augengegend.

16. (31. VIII. 1920.)

Die Haut wird faradisch gereizt. Die ursprüngliche Blaufärbung wird braun. Jedoch tritt die Reaktion viel später ein wie die Aufhellung der Scholle bei fa- 
radischer Reizung. Die Braunfärbung, die zuerst an der Elektrodenstelle lokalisiert bleibt, breitet sich über größere Hautstrecken aus. Während durch Faradisieren bei Pleuronectes sich die ganze Haut aufhellt, ist dies bei Cyclopterus nicht der Fall.

21. (2. IX. 1920.)

Gehirn und Medulla oblongata wurden freigelegt. Es wurde faradisch gereizt die Lob. olfact., Opticus, Groß-, Zwischen- und Kleinhirn. Bei Reizung der

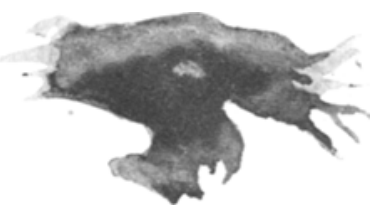

Abb. 16. Chromatophore aus der oberen Hautschicht. Medulla trat eine schwache Aufhellung (Rosafärbung) ein. Die geringe Intensität des Farbwechsels mag wohl auf der durch die Operation und Blutverlust bedingten Schädigung zurückzuführen sein, da diese Tiere besonders empfindlich sind.

17. (31. VIII. - 1920.)

Bei Strychninvergiftung wurde die Färbung bei Eintritt der Krämpfe und Erregbarkeitssteigerung hellrosa. Die schwarzen Chromatophoren waren also in Ballung. (Strychnin wurde dem Wasser des Aquariums zugesetzt.) Weitere Giftversuche konnten nicht mehr gemacht werden.

Die Chromatophoren von Cyclopterus reagieren a elektrischen Reiz.

Siekönnen durch Reizung der Medulla oblongata zur Kontraktion gebrachtwerden. Also liegt auch hier das Chromatophorenzentrum, wie bei allen bis jetzt unter. suchten Fischarten, im Nachhirn (Medulla oblongata).

Bemerkung zu den Textabbildungen.

Chromatophorenvergrößerung: Leitz' Ölimmers. 1/12, Okular 0; Entfernung zwischen Zeichenprisma and Zeichentisch $40 \mathrm{~cm}$. Bei der Reproduktion auf $3 / 4$ ver kleinert. Die Abbildungen 1 und 7 sind dem „Führer durch das Aquarium der Zoologischen Station Büsum“ entnommen. 